\title{
Near infrared imaging of the broad absorption line quasar BAL QSO 0134+3253^
}

\author{
J. Zuther and A. Eckart
}

\author{
I. Physikalisches Institut, Universität zu Köln, Zülpicher Str. 77, 50937 Köln, Germany \\ e-mail: zuther@ph1.uni-koeln.de
}

Received 8 March 2005 / Accepted 5 August 2005

\section{ABSTRACT}

In this paper we present near infrared (NIR) imaging data of the host galaxy of the broad absorption line quasar (BALQ) at $z=2.169$, serendipitously found close to 3C 48 (Canalizo et al. 1998, AJ, 115, 890). The data were obtained with the ESO-VLT camera ISAAC during period 67. We find extended, rest-frame optical emission around the BALQ after subtracting a scaled stellar point spread function from the quasar nucleus in $J, H$, and $K$ s. The extended rest-frame optical emission can be interpreted as an approximately 2 Gyr old stellar population composing the host galaxy of the BALQ or a stellar population of similar age associated with an intermediate $(z=1.667)$ absorption system spectroscopically identified by Canalizo et al. (1998). The rest-frame-UV emission on the other hand is dominated by a young, 500 Myr old stellar population (Canalizo et al. 1998). The UV/optical colors resemble a mixture of the two populations, of which the young one accounts for about $80 \%$. Assuming that the residual emission is located at the BALQ redshift, we find that the host galaxy has a resolved flux of about $10 \%$ of the BALQ flux. The physical scale is quite compact, typical for radio quiet QSOs or Lyman break galaxies at these redshifts, indicating that the systems are still in the process of forming.

Key words. galaxies: fundamental parameters - galaxies: high-redshift - infrared: galaxies - galaxies: active

\section{Introduction}

There is evidence for an intimate link between the growth of massive black holes and the galaxy bulges they reside in (e.g. Page et al. 2001). This implies the existence of physical feedback mechanisms to regulate their coeval evolution. In the quasar phase, i.e. during the bulk of the accretion of matter onto the central massive black hole, winds/outflows from the innermost region of the quasar can be an important source of such a feedback (e.g. Gallagher et al. 2004).

Broad absorption line quasars (BALQs) form a rare class, comprising about $20 \%$ of the QSO population at moderate to high redshifts (Hewett \& Foltz 2003). The BAL phenomenon is related to outflows in quasars (e.g. Hamann \& Sabra 2004). Such outflows constitute metal absorption systems with large blueshifted velocities (several thousand $\mathrm{km} \mathrm{s}^{-1}$ ). There are currently two standard interpretations of these phenomena. (1) Unified scheme: under the assumption of the unified scheme for active galaxies (Antonucci 1993), the absorbing clouds have a small covering factor as seen from the QSO nucleus (Weymann et al. 1991). Thus, the frequency of detection just translates to the rate at which our line of site intercepts the outflow. (2) Evolutionary scheme: a different interpretation is that these objects are very young and are ejecting their gaseous envelopes at very high velocities following the initial turn-on of the active galactic nucleus (Hazard et al. 1984; Briggs et al. 1984).

\footnotetext{
* Based on observations with the VLT; proposal number 67.B-0019.
}

The unified scheme would imply that all radio-quiet QSOs should be classified as BALQs if observed from the proper angle. This was supported until recently by the work of (Stocke et al. 1992). However, in a spectroscopic follow-up of radio selected BALQs, Becker et al. (2000) find a population of FIRST $^{1}$ BALQs being rather radio-intermediate, indicating that the radio properties do not support the "simple" scenario stated above.

On the other hand, in case of the evolutionary scenario an enhanced sub-mm radiation would be expected, because the BALQs still possess a lot of molecular gas and dust while actively forming stars (Hall et al. 1997; Reichard et al. 2003; Richards et al. 2003) and the host galaxies would appear to be at early stages in their evolution. There seems to be a subclass of BALQs with enhanced far-IR emission (Low et al. 1989) for which this could be the case. The large number of lowionization BALQs in IRAS-selected luminous QSOs, along with the reddening from dust in these objects, suggests that this subclass may represent an evolutionary transition between ultraluminous IR galaxies and the classical QSO population in the early universe (Lipari 1994; Voit et al. 1993; Egami et al. 1996). Recent studies of BALQs by Lewis et al. (2003) and Willott et al. (2003), however, show no differences in the sub$\mathrm{mm}$ properties between BALQs and non-BALQs which supports a small BAL covering factor.

\footnotetext{
1 VLA Faint Images of the Radio Sky at Twenty-centimeters.
} 
Table 1. Previous measurements of the BALQ and the extended emission.

\begin{tabular}{ccc}
\hline \hline Filter & Total & Host \\
$(1)$ & $(2)$ & $(3)$ \\
\hline$g$ & $21.2^{a}$ & - \\
$F 555 W=V$ & $21.14^{b}$ & $23.8 \pm 0.1^{b}$ \\
$F 814 W=I$ & $21.28^{b}$ & $24.1 \pm 0.1^{b}$ \\
$H+K$ & - & $5 \times 10^{-20} \mathrm{erg} \mathrm{s}^{-1} \mathrm{~cm}^{-2} \AA^{-1} \operatorname{arcsec}^{-2 b, c}$ \\
\hline
\end{tabular}

${ }^{a}$ Kirhakos et al. (1994); listed as a star.

${ }^{b}$ CS98; measured in a 2". 6 diameter aperture.

${ }^{c}$ CS98; $1 \sigma$ detection threshold.

In addition Archibald et al. (2002) show that the star forming phase of the host and the dust/gas rich BAL wind do not need to coincide in time, thus providing constraints on alternative evolutionary models.

As the detailed nature of these objects is still under debate, the study of their host galaxies could provide important complementary information.

One recent example of a detected extended emission around a BAL QSO is the object 0134+3253. (Canalizo et al. 1998, hereafter CS98) present in their work a rest frame UV spectrum as well as Hubble Space Telescope (HST) rest frame UV/optical images of the $z=2.169$ BALQ 0134+3253. The authors find a deep absorption trough blueward of the C IV line which is composed of three elements and wind velocities of up to $-12000 \mathrm{~km} \mathrm{~s}^{-1}$. The red edge is about $+1000 \mathrm{~km} \mathrm{~s}^{-1}$ (their Fig. 2). There is no more spectroscopic information available to further classify this QSO as an high- or low-ionization BALQ. From their optical spectrum an intermediate absorption system at $z \approx 1.667$ is evident, probably being a damped Ly $\alpha$ absorber (CS98). In this context it is not clear whether the redness with respect to the average HST quasar spectrum (Zheng et al. 1997) is due to the absorption system or intrinsic to the quasar host galaxy. Photometric data are summarized in Table 1. The HST $F 555 W$ and $F 814 W$ filters approximately correspond to $V$ and $I$ band, respectively, whereas $g$ lies between $B$ and $V$.

In Sect. 2 we will briefly describe the ISAAC observations. In Sect. 3 we will present rest frame optical photometry of this BALQ and its extended emission to complement the rest frame UV data of CS98. Unless noted, we use a cosmology with $H_{0}=$ $70 \mathrm{~km} \mathrm{~s}^{-1} \mathrm{Mpc}^{-1}, \Omega_{\mathrm{m}}=0.3$, and $\Omega_{\Lambda}=0.7$ (Spergel et al. 2003) throughout this paper.

\section{Observation and data reduction}

The data were acquired with the Infrared Spectrometer and Array Camera (ISAAC, Moorwood 1995) mounted at the Nasmyth B focus of Unit Telescope 1 (UT1, Antu) of the Very Large Telescope (VLT; ESO, Chile).

Imaging was performed in the broad bands $J, H$ and $K \mathrm{~s}$ $(1.2-2.2 \mu \mathrm{m})$. The $1024 \times 1024$ pixel Hawaii Rockwell array detector provides a pixel scale of $0.1484^{\prime \prime} /$ pixel with a field of view of $152 \times 152 \mathrm{arcsec}^{2}$. On source integration time was $2880 \mathrm{~s}, 2160 \mathrm{~s}$, and $1080 \mathrm{~s}$ in $J, H$, and $K \mathrm{~s}$ respectively. The data were reduced with IRAF using standard procedures. In all bands the images seem to be adequately flat with a variation smaller than $3 \%$ over the area of interest. Additionally, the science object was moved across the array, so that these variations are reduced due to the averaging of the individual exposures. Successive object and sky observations were subtracted and shifted to produce sky subtracted and averaged (median) images. Figure 1 shows the $H$-band image including the quasar $3 \mathrm{C} 48$. The average seeing was about $F W H M \approx 0 . ' 4$ in $H$ and about 0.5 in $J$ and $K$ s. The calibration of our data relies on the observation of standard stars (Hawarden et al. 2001; Persson et al. 1998). More details on the data reduction can be found in Zuther et al. (2004).

\section{Results and discussion}

While the connection between the super massive black holes $(\mathrm{SMBH})$ and their host galaxies is still not clearly understood, the host galaxies are extremely difficult to observe at redshifts $z>1$, because of the high contrast between nucleus and host galaxy and the surface brightness dimming. The high sensitivity of the VLT and its instrumentation as well as the good seeing conditions $(F W H M<0$.'5) enabled us to investigate the extended rest-frame optical emission around the BALQ $0134+3252$, providing a view on the unobscured star formation in these galaxies.

We measure fluxes in $J, H$, and $K$ s of the BALQ, the potential host galaxy and the nucleus. We do not correct for Galactic extinction, because its values are smaller than the photometric error. Our results are listed Table 2. Previous measurements by Kirhakos et al. (1994) and CS98 are given in Table 1.

\subsection{Overall properties}

Measuring the fluxes in a 2 ". 6 diameter aperture centered on the quasar nucleus (as done by CS98), we find the overall colors of the BALQ to be $J-H=0.8 \pm 0.1$ and $H-K=$ $0.5 \pm 0.1$ (cf. Fig. 5). Using the $F 555 W$ and the $g$ magnitude together with the transformation from $g$ to $B$ magnitudes $(g=V-0.19+0.41[B-V])$ stated in Kirhakos et al. (1994), we get an observed $B-K \approx 3.3$. This is only slightly reddened compared to a typical $B-K=3$ for unreddened $z>1$ quasars (Hall et al. 1997, and references therein). CS98 found a small reddening of the rest-frame UV spectrum of the BALQ compared to the HST composite quasar spectrum (Zheng et al. 1997). Estimating this reddening to be about a factor of 1.3 and following the UV-optical extinction law (e.g. Sofia et al. 2005), the small UV-reddening contributes only little to the rest-frame optical, resulting in the slightly reddened observed $B-K$ color.

In the untreated $H$-band image (Fig. 1) a non stellar-like shape of the BALQ can already be emphasized, which we will discuss next.

\subsection{Radial profiles and 2-dimensional images}

In order to get an improved impression of the faint host galaxy emission we extracted two radial profiles orthogonal to each other in each band, with one along the primary extended component at a position angle (PA) of about $45^{\circ}$. The profiles are 


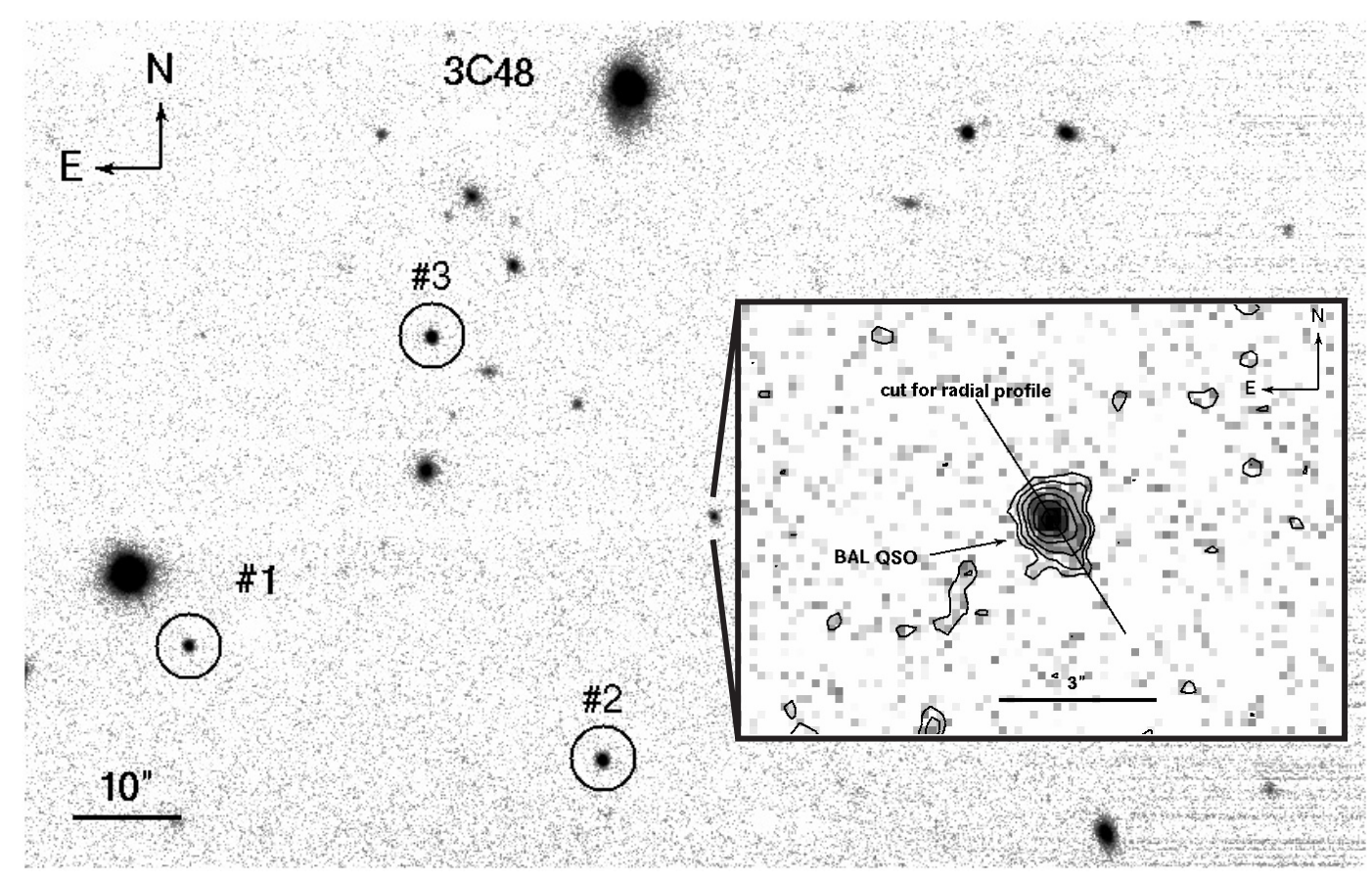

Fig. 1. $H$ band ISAAC image of the field of 3C 48 (Zuther et al. 2004). The inset shows our $H$ band close up of the broad absorption line quasar. The lowest contour is at the level of sky noise. Also indicated is the primary cut used for the radial profiles. The stars used for PSF estimation are marked with (\#1, \#2, and \#3). See text for details.

Table 2. Photometry of the BALQ and the extended emission. The first part lists $J, H$, and $K$ s magnitudes measured from the radial profiles and in a 1".5 diameter aperture. The second part lists the deduced colors. Total fluxes and colors are listed in Cols. (2) and (3). Columns (4) and (5) give the host magnitudes and colors, and Cols. (6) and (7) correspondingly for the subtracted nucleus.

\begin{tabular}{ccccccc}
\hline \hline Filter & \multicolumn{2}{c}{ Total } & \multicolumn{2}{c}{ Host } & \multicolumn{2}{c}{ Nucleus } \\
& \multicolumn{2}{c}{$[\mathrm{mag}]$} & \multicolumn{2}{c}{$[\mathrm{mag}]$} & \multicolumn{2}{c}{$[\mathrm{mag}]$} \\
& rad. prof. & $1^{\prime \prime} 5$ ap. & rad. prof. & $11^{\prime \prime} 5$ ap. & rad. prof. & $11^{\prime \prime} 5 \mathrm{ap}$. \\
$(1)$ & $(2)$ & $(3)$ & $(4)$ & $(5)$ & $(6)$ & $(7)$ \\
\hline$J$ & $19.9 \pm 0.1$ & $20.2 \pm 0.1$ & $23.5 \pm 0.2$ & $22.6 \pm 0.1$ & $22.1 \pm 0.2$ & $20.3 \pm 0.1$ \\
$H$ & $19.1 \pm 0.1$ & $19.4 \pm 0.1$ & $22.4 \pm 0.1$ & $21.4 \pm 0.1$ & $21.2 \pm 0.1$ & $19.5 \pm 0.1$ \\
$K \mathrm{~s}$ & $18.5 \pm 0.1$ & $18.9 \pm 0.1$ & $21.8 \pm 0.2$ & $20.7 \pm 0.1$ & $20.6 \pm 0.2$ & $19.1 \pm 0.1$ \\
\hline \multicolumn{7}{c}{ Colors } \\
\hline$J-H$ & $0.8 \pm 0.1$ & $0.8 \pm 0.1$ & $1.1 \pm 0.2$ & $1.2 \pm 0.1$ & $0.9 \pm 0.2$ & $0.8 \pm 0.1$ \\
$H-K \mathrm{~s}$ & $0.6 \pm 0.1$ & $0.5 \pm 0.1$ & $0.6 \pm 0.2$ & $0.7 \pm 0.1$ & $0.6 \pm 0.2$ & $0.4 \pm 0.1$ \\
$B-K \mathrm{~s}$ & 3.2 & - & - & - & - & - \\
\hline
\end{tabular}

measured over cone-like sections with a cone angle of $60^{\circ}$ along this extension (apparent major axis) and along the orthogonal axis at $\mathrm{PA}=132^{\circ}$ (cf. Fig. 1).

The radial profiles of the BALQ are compared with the profiles of three nearby unsaturated stars, circular in appearance (\#1, \#2, and \#3 in Fig. 1), where the stellar profiles are scaled to the QSO peak flux (Figs. 2a,c, e,g, and i,j). It is evident from the radial profiles that the intensity distribution in $J$ and $H$ is extended compared to the stellar radial profiles. Next, we fitted a Moffat function, which well represents the ISAAC seeing-limited PSF, to each stellar profile. After subtracting the three functions from the galaxy profile individually, a residual emission is clearly visible in the $J$ and $H$ band (Figs. 2b,d and $\mathrm{f}, \mathrm{h}$ ), whereas in $K$ s there is only a faint and noisy residual profile (Fig. 2j) visible. The low signal-to-noise ratio (SNR) in the $K$ s-band residual (especially in the PA $=45^{\circ}$ cone section (cf. Fig. 3)) can be accounted for by the facts, that young stellar populations are fading towards longer rest-frame optical wavelengths (e.g. Fig. 8 of Jahnke et al. 2004a) and that the sky background increases towards the observed $K$ s. In favor of clarity for the reader we only show the error bars of the "middle" profile (star \#3) which we also used to estimate the flux. The stellar flux was scaled such that its subtraction results in a smooth and flat central part of the profile (cf. discussion in McLeod \& McLeod 2001). This presents a conservative lower limit for the potential emission of a host galaxy. A subtraction of a larger point-like contribution resulting in a decrease of the intensity towards the center is likely to be not physical, and would yield colors not consistent with those of normal galaxies.

We measure the fluxes within the two perpendicular conesections by summing over the profiles. Then we calculate the mean flux as the geometric mean of the two measurements, 

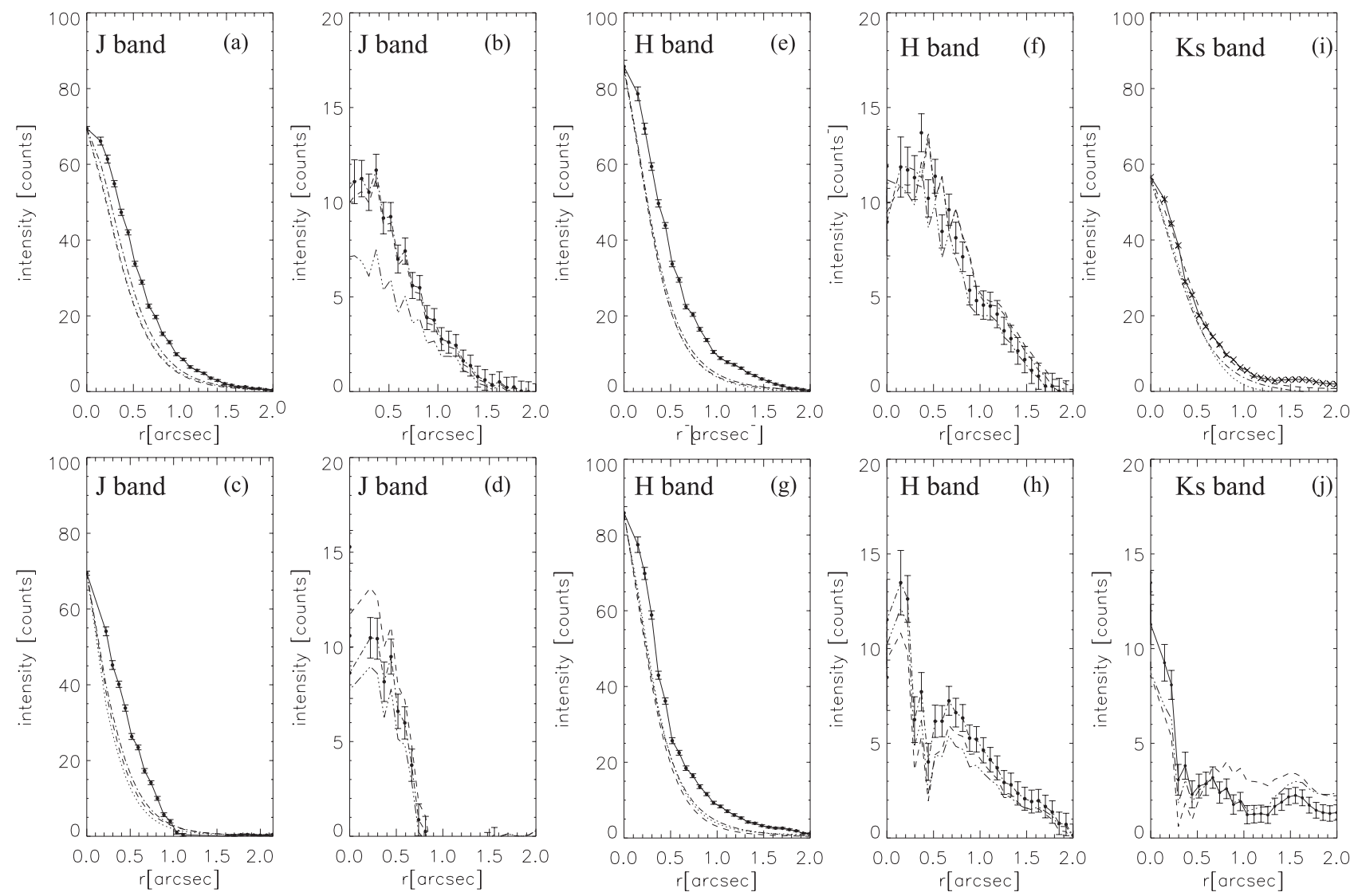

Fig. 2. a) $J$ band radial profile of the BALQ (solid line) and three nearby stars (dotted, dashed, dash-dot; see Fig. 1). The peak flux of each star is scaled to the peak flux of the BALQ. b) The residual radial host galaxy profile after subtracting the stellar profile, scaled such that the residual emission is approximately flat close to the center. c) and d) correspond to a) and b) but for a cut along a position angle of $132^{\circ}$.e), f) and g), h) same as a), b) and c), d) but for $H$ band. i) is the $K$ s band radial profile for the position angle of $132^{\circ}$ and j) is the residual after subtraction of the stellar profile. The profile of the BALQ along the PA of $45^{\circ}$ shows only a marginal extent with respect to the stellar profile. See text for details.

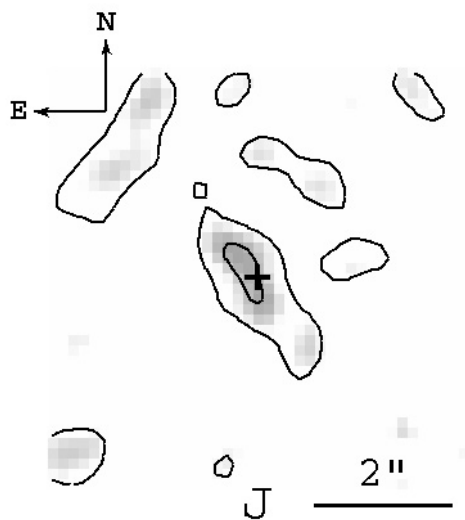

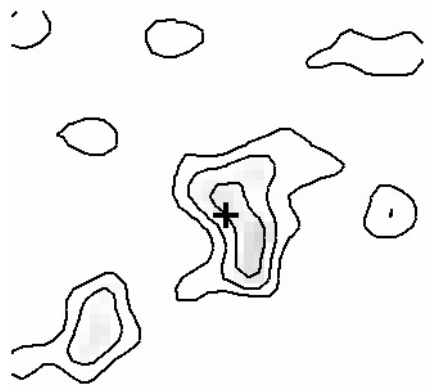

$\mathrm{H}$

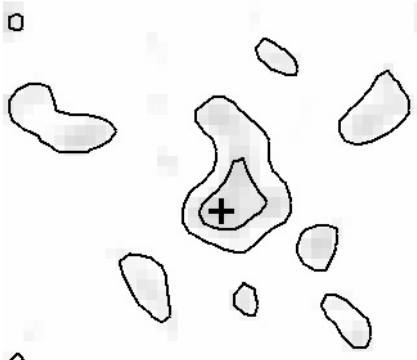

J Ks

Fig. 3. PSF-subtracted $J, H$, and $K$ s images. After subtraction the images have been smoothed with a three pixel Gaussian. The contour levels correspond to $(1,4) \times \sigma$ in $J,(1.5,3,4) \times \sigma$ in $H$, and $(2,3) \times \sigma$ in $K$ s of the sky noise. The crosses show the position of the nucleus from which the PSF is subtracted.

because assuming an elliptical morphology we would overestimate the flux when using the major axis alone, and underestimate the flux when only considering the minor axis. The integrated fluxes in our $H$ and $K$ s measurements are consistent with the limits given by CS98. From the residual fluxes we can estimate colors of the extended emission around the BALQ.
Similarly, we use the same star (\#3) and scaling for the subtraction of the nucleus in the 2-dimensional (2D) images. The results were smoothed with a 3 pixel FWHM Gaussian in order to reduce the noise. Nevertheless, the images show quite strong fluctuation, whereas the $H$ band image has the best signal-to-noise ratio. We measure the fluxes in 


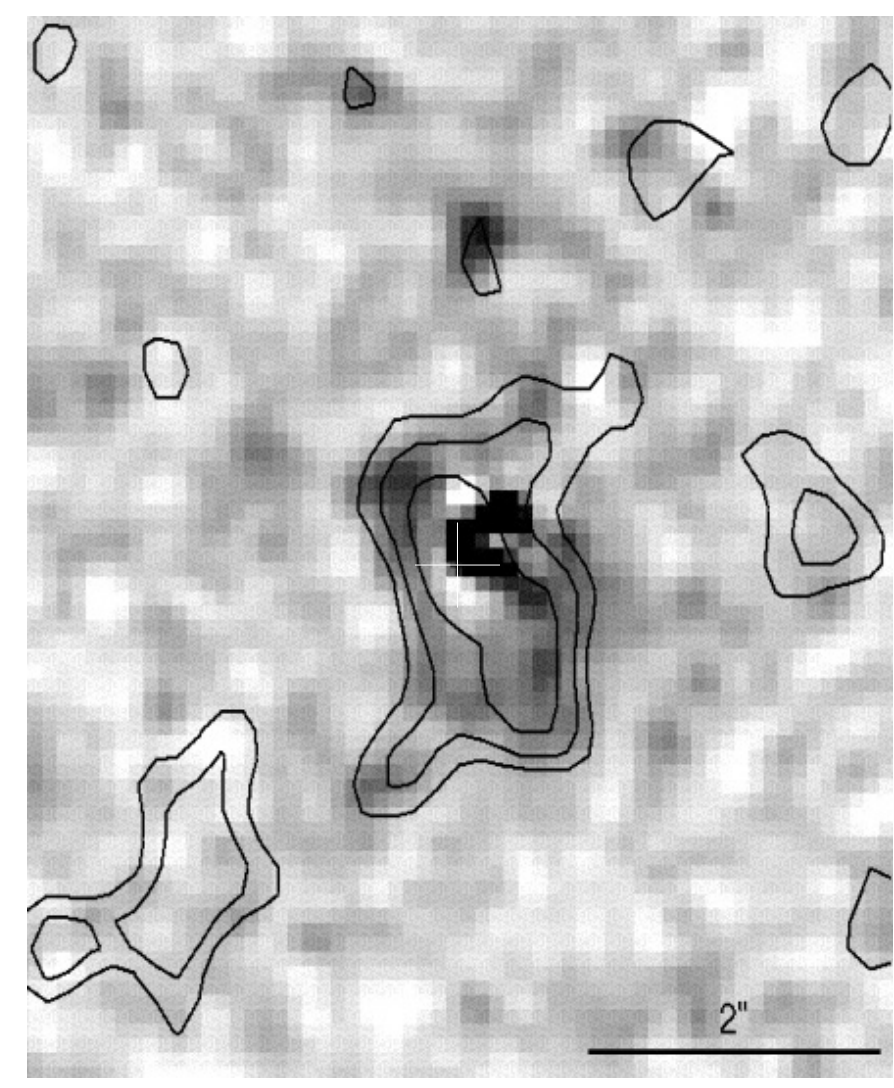

Fig. 4. PSF-subtracted HST $F 814 W$ image from CS98 overlaid with contours of our three-pixel-smoothed, PSF-subtracted $H$ band image. The lowest contour level corresponds to $1.5 \times \sigma$ of the sky noise. The white cross shows the position of the nucleus in our $H$ band image. North is up and east is to the right. Note that the PSF-subtracted HST images is extracted from CS98. Despite the values given in Table 2 no further photometric information is available.

1 .'5 diameter apertures, because up to this size the fluctuations are not significant. The given errors only include measurement uncertainties. The host contribution to the total light in the NIR is of the order of $10 \%$. The PSF subtracted images presented in Fig. 3 show an overall elliptical morphology. The lowest contours correspond to $\sim 2 \sigma$ of the sky noise. The structures at distances larger than $3^{\prime \prime}$ from the nucleus are most probably due to noise. The feature about $2^{\prime \prime}$ southeast of the nucleus ( $H$ and $K \mathrm{~s}$ ) is also visible in the untreated image (inset in Fig. 1) although it is not clear whether it is connected to the BALQ at all. Fig. 4 shows our PSF-subtracted $H$ band image in contours overlaid on the PSF-subtracted HST $V$-band image extracted from CS98. Although the exact position of the nucleus in the HST image is not known to us, the overall size and orientation is similar to what is reported on the optical by CS98.

The colors of the BALQ, its host, and its nucleus together with colors of starbursts of different ages at $z=2$ and $z=1.7$ are presented in Fig. 5. For comparison colors of other BALQs listed in Hall et al. (1997) are displayed. Among them are Hawaii 167 (Cowie et al. 1994) and the radioloud QSO 1556+3517 (Hall et al. 1997), two of the reddest $(B-K>5)$ quasars known. Within the errors the colors of
Table 3. Physical scales of the residual emission determined from the $H$-band radial profile.

\begin{tabular}{ccccc}
\hline \hline$H W H M_{\text {major }}$ & $H W H M_{\text {minor }}$ & $r_{1 / 2}$ & {$\left[\mathrm{kpc} /{ }^{\prime \prime}\right]$} & {$\left[\mathrm{kpc} /{ }^{\prime \prime}\right]$} \\
{$\left[{ }^{\prime}\right]$} & {$\left[{ }^{\prime \prime}\right]$} & {$\left[{ }^{\prime \prime}\right]$} & $(z=2)$ & $(z=1.7)$ \\
$(1)$ & $(2)$ & $(3)$ & $(4)$ & $(5)$ \\
\hline $0.9 \pm 0.1$ & $0.5 \pm 0.3$ & $0.4 \pm 0.1$ & 8.3 & 8.5 \\
\hline
\end{tabular}

the two methods (radial profiles and 2D photometry) are consistent with each other.

Combining the available photometric data, we use the four colors $(F 555 W-F 814 W),(F 814 W-J),(J-H)$, and $(H-K$ s $)$ to get a first impression of the stellar content of the extended emission by comparison with colors of simple stellar populations (Figs. 5 and 6). We calculated colors of a 1 Gyr starburst followed by passive evolution at redshifts of $z=2$ and $z=1.7$ using the population synthesis code of Bruzual \& Charlot (2003), and utilizing the Padova1994 initial spectral energy distributions of solar metalicity with a Chabrier initial mass function (Chabrier 2003).

The host colors from Fig. 5 infer a stellar population of about 2 Gyr dominating the emission at the redshift of the BALQ. At the redshift of the intermediate absorber, the ages are somewhat older. Thus, we see an older stellar population in the rest-frame optical than did CS98 in the rest-frame UV only, which is typically dominated by young and hot stars. The view on the unobscured star formation can be seen in Fig. 6. The rest-frame UV/optical host colors lie well below the color track of the 1 Gyr starburst. We calculated the mixing curve for a composite color made up of a 0.1 or $0.5 \mathrm{Gyr}$ and a 2 Gyr population (the two thick dotted curves in Fig. 6). It is evident that a young population dominates the rest-frame UV/optical emission, accounting for about $80 \%$ of the colors. Correspondingly, the dominating population would be somewhat younger at $z=1.667$. Similar results are found by Jahnke et al. (2004b, their Fig. 6) in their study of likewise luminous $z \sim 2-3$ GEMS quasar hosts.

Further details on the stellar populations are difficult to obtain, because of the possible degeneracies of colors related to dust and metalicity (cf. Jahnke et al. 2004b). Moreover, the noisy residual after subtraction of the QSO nucleus can influence the resulting colors.

Assuming that the extended emission originates at the redshift of the BALQ, $z=2.169,1^{\prime \prime}$ corresponds to a linear scale of $8.3 \mathrm{kpc}$. In the unified scheme BALQs should be common QSOs at the respective redshift, viewed such that our line of sight intercepts the QSO wind. Thus, we can compare our results with hosts of present day and $z \sim 2-3$ radio-loud and radio-quiet QSOs. For this purpose we use two model independent measures of the host galaxy size (Table 3). First we measure the mean half width at half maximum (HWHM) of the radial profile, because there is some degree of degeneracy in the radial profiles (cf. Fig. 2h). Using the $H$ band profile, we find a $H W H M$ radius of $r=00^{\prime} 9 \pm 00^{\prime} 1$, i.e. a physical scale of $(7.5 \pm 0.8) \mathrm{kpc}$, along the major axis. For the minor axis we find $r=0.5 \pm 0.3$, corresponding to $(4.2 \pm 2.5) \mathrm{kpc}$ along the minor axis. In case of an elliptical galaxy this would give a Hubble 


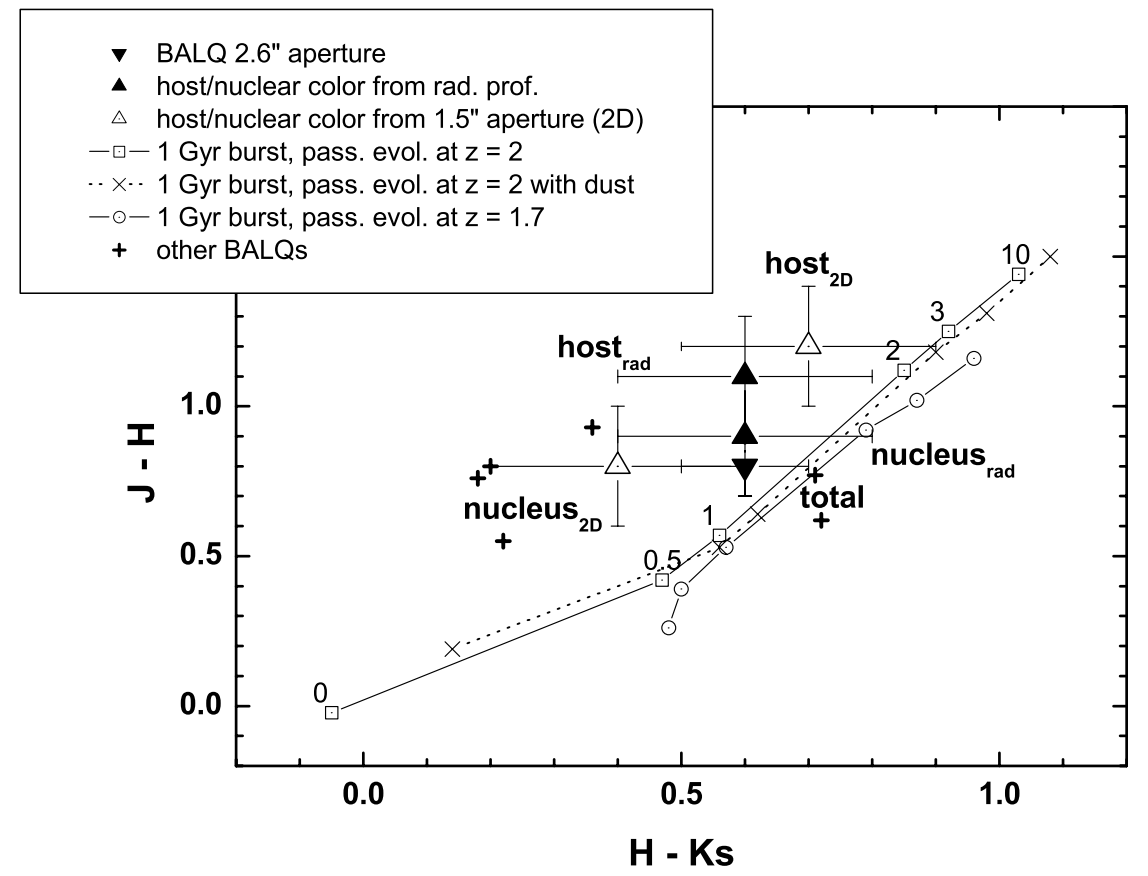

Fig. 5. Two-color diagram presenting the $(J-H)$ vs. $(H-K \mathrm{~s})$ observed colors of the BALQ (upside-down triangle), the host, and the nucleus (filled triangles) as discussed in the text. The hollow triangles represent the host and nuclear colors measured in a 1". 5 diameter aperture from the 2D PSF-subtracted images. Also shown are colors of BALQs (crosses) from Hall et al. (1997). For comparison colors of a $1 \mathrm{Gyr}$ starburst followed by passive evolution for ages of $0,0.5,1,2$, 3 , and $10 \mathrm{Gyr}$ at redshifts $z=2$ (open rectangles) and $z=1.7$ (open circles) are displayed (using Bruzual \& Charlot 2003). The dotted line shows the effect of reddening of the $z=2$ track following the model of Charlot \& Fall (2000) with $\tau_{V}=1.5$ and $\mu=0.3$.

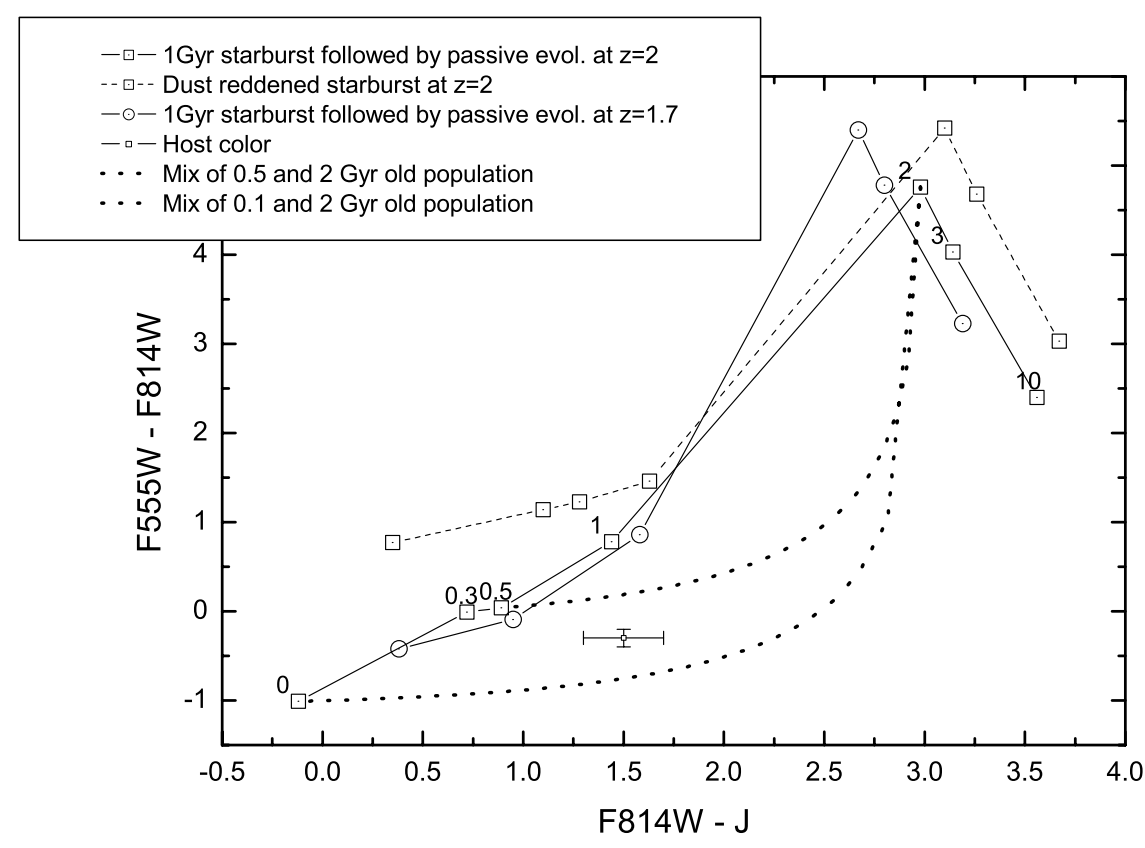

Fig. 6. Two-color diagram presenting the $(F 555 W-F 814 W)$ vs. $(F 814 W-J)$ observed colors of the BALQ host galaxy (cf. Fig. 5). Also shown are the colors of $1 \mathrm{Gyr}$ single starbursts followed by passive evolution at $z=2$ (open squares; numbers indicate the age in Gyr) and $z=1.7$ (open circles). The effect of reddening of the $z=2$ track, with $\tau_{V}=1.5$ and $\mu=0.3$ according to the model of Charlot \& Fall (2000), is shown as open squares joined by a dashed line. Furthermore two curves displaying the effect of mixing of a 0.1 Gyr and 2 Gyr old (lower dotted line) and of a 0.5 Gyr and 2 Gyr old (upper dotted line) population are shown.

type E4. For a spiral galaxy this would imply an inclination of about $(60 \pm 10)^{\circ}$. The axis ratio is also in agreement with the 2D PSF-subtracted $H$ band image (Fig. 4). As a second measure, the half-light radius derived from the $H$ band radial profile is about $r_{1 / 2}=0 \prime^{\prime} 4 \pm 0{ }^{\prime}{ }^{\prime} 1$, corresponding to $(3.3 \pm 0.8) \mathrm{kpc}$. Ridgway et al. (2001) find a similar physical scale $(2.3 \mathrm{kpc})$ in their sample of radio quiet QSOs at comparable redshift. These quite compact sizes seem to be more typical for Lyman break galaxies at $z \sim 2-3$ (Giavalisco et al. 1996; Dickinson 2000) than for present day QSOs hosts (McLure et al. 1999; Bahcall et al. 1997). This could imply that the compact systems are still in the process of forming. Note that because of the low SNR of our data we could be biased towards more compact emission. Furthermore we use the method described in
Jahnke et al. (2004b) to estimate an absolute $B$ band magnitude (Table 4). Assuming an optical power law, $f_{\lambda} \propto \lambda^{\beta}$, we can find the index $\beta$ from the NIR colors and then calculate the apparent $B$ magnitude. We find $M_{B} \approx-22.6 \pm 0.2$, which is also comparable to the results of Ridgway et al. (2001). The possible host would correspond to a $\sim 4 \times L_{B}^{*}$ galaxy using the luminosity function of local galaxies from Loveday et al. $(1992)^{2}$. This is at the upper end of the range of luminosities $\left(0.2-4 L_{B}^{*}\right)$ found by Ridgway et al. (2001).

At the redshift of the absorber, $z=1.667,1^{\prime \prime}$ corresponds to $8.5 \mathrm{kpc}$. Thus, the physical scales would correspond to

2 Their $M_{B}^{*}=-19.5$ for $h=1$ has been adapted to our cosmology, i.e. $M_{B}^{*} \approx-21$. 
Table 4. Estimated absolute $B$-band magnitude from 1".5 aperture measurement. Given are the values for the redshift of the BALQ $(z=2.169)$ and the redshift of the intermediate absorber $(z=1.667)$. See text for details.

\begin{tabular}{cccc}
\hline \hline \multicolumn{2}{c}{$M_{B}$ Host } & \multicolumn{2}{c}{$M_{B}$ Nucleus } \\
$(z=2.169)$ & $(z=1.667)$ & $(z=2.169)$ & $(z=1.667)$ \\
$(1)$ & $(2)$ & $(3)$ & $(4)$ \\
\hline$-22.5 \pm 0.2$ & $-21.9 \pm 0.2$ & $-24.9 \pm 0.2$ & $-24.2 \pm 0.2$ \\
\hline
\end{tabular}

$H W H M_{\text {major }} \approx 7.7 \mathrm{kpc}$ and $H W H M_{\text {minor }} \approx 4.3 \mathrm{kpc}$. The halflight radius would be $r_{1 / 2} \approx 3.4 \mathrm{kpc}$. The absolute $B$ magnitude of $M_{B} \approx-21.9$ would correspond to a $2.5 \times L_{B}^{*}$ host galaxy. Together with the colors this is still consistent with a relatively young stellar population at moderate extinction and with the host sizes at these redshifts. We therefore cannot pinpoint, whether the emission is coming from the BALQ host or from the intermediate absorber.

\section{Summary}

We presented rest-frame optical extended emission around the BALQ $0134+3252$, complementing previous results on the rest-frame UV extended emission (CS98). The UV/optical colors indicate a mixture of a young $\$ 500 \mathrm{Myr}$ and a further evolved $\sim 2$ Gyr old stellar population. As the broadband data are affected by various degeneracies related to dust and metalicities, a reliable age dating is still difficult to accomplish. This is even complicated due to the QSO subtraction in our noisy data. The rest-frame optical morphology is similar to the UV morphology. If associated with the BALQ, the host galaxy would correspond to a present day $4 L_{B}^{*}$ host. Its compact size, $r_{1 / 2}=3.2 \mathrm{kpc}$, is comparable to radio quiet QSOs and Lyman break galaxies at $z \sim 2-3$, but smaller than present day QSO hosts. The small size, the influence of a young stellar population, and the high luminosity fit well into the framework of hierarchical galaxy formation. At the redshift of the potential Ly $\alpha$ absorber, the sizes are somewhat larger and the luminosity would correspond to a present day $2.5 L_{B}^{*}$ host. Also the rest-frame optical stellar populations would be evolved slightly more than at the BALQ redshift. The data are still comparable to results found for RQQs and LBGs. We cannot, however, distinguish between the BALQ or Ly $\alpha$ absorber origin of the extended continuum emission.

A more detailed stellar population analysis awaits higher SNR and further spectroscopic observations. These can also help uncovering the yet unclear source of the UV/optical emission.

Acknowledgements. We would like to thank Julia Scharwächter, Rainer Schödel, Sebastian Fischer and Thomas Bertram for fruitful discussions. The comments and suggestions of the anonymous referee were very helpful in revising this paper. This work was supported in part by the Deutsche Forschungsgemeinschaft (DFG) via grant SFB 494.

\section{References}

Antonucci, R. 1993, ARA\&A, 31, 473

Archibald, E. N., Dunlop, J. S., Jimenez, R., et al. 2002, MNRAS, 336, 353

Bahcall, J. N., Kirhakos, S., Saxe, D. H., \& Schneider, D. P. 1997, ApJ, 479, 642

Becker, R. H., White, R. L., Gregg, M. D., et al. 2000, ApJ, 538, 72

Briggs, F. H., Turnshek, D. A., \& Wolfe, A. M. 1984, ApJ, 287, 549

Bruzual, G., \& Charlot, S. 2003, MNRAS, 344, 1000

Canalizo, G., Stockton, A., \& Roth, K. C. 1998, AJ, 115, 890 (CS98)

Chabrier, G. 2003, PASP, 115, 763

Charlot, S., \& Fall, S. M. 2000, ApJ, 539, 718

Cowie, L. L., Songaila, A., Hu, E. M., et al. 1994, ApJ, 432, L83

Dickinson, M. 2000, in Astronomy, physics and chemistry of $\mathrm{H}_{3}^{+}, 2001$

Egami, E., Iwamuro, F., Maihara, T., Oya, S., \& Cowie, L. L. 1996, AJ, 112, 73

Gallagher, S. C., Brandt, W. N., Chartas, G., Garmire, G. P., \& Sambruna, R. M. 2004, Adv. Space Res., 34, 2594

Giavalisco, M., Steidel, C. C., \& Macchetto, F. D. 1996, ApJ, 470, 189

Hall, P. B., Martini, P., Depoy, D. L., \& Gatley, I. 1997, ApJ, 484, L17

Hamann, F., \& Sabra, B. 2004, in ASP Conf. Ser., 203

Hawarden, T. G., Leggett, S. K., Letawsky, M. B., Ballantyne, D. R., \& Casali, M. M. 2001, MNRAS, 325, 563

Hazard, C., Morton, D. C., Terlevich, R., \& McMahon, R. 1984, ApJ, 282,33

Hewett, P. C., \& Foltz, C. B. 2003, AJ, 125, 1784

Jahnke, K., Kuhlbrodt, B., \& Wisotzki, L. 2004a, MNRAS, 352, 399

Jahnke, K., Sánchez, S. F., Wisotzki, L., et al. 2004b, ApJ, 614, 568

Kirhakos, S., Sargent, W. L. W., Schneider, D. P., et al. 1994, PASP, 106, 646

Lewis, G. F., Chapman, S. C., \& Kuncic, Z. 2003, ApJ, 596, L35

Lipari, S. 1994, ApJ, 436, 102

Loveday, J., Peterson, B. A., Efstathiou, G., \& Maddox, S. J. 1992, ApJ, 390, 338

Low, F. J., Cutri, R. M., Kleinmann, S. G., \& Huchra, J. P. 1989, ApJ, 340, L1

McLeod, K. K., \& McLeod, B. A. 2001, ApJ, 546, 782

McLure, R. J., Kukula, M. J., Dunlop, J. S., et al. 1999, MNRAS, 308, 377

Moorwood, A. F. 1995, in Infrared Detectors and Instrumentation for Astronomy, ed. A. M. Fowler, Proc. SPIE, 2475, 262

Page, M. J., Stevens, J. A., Mittaz, J. P. D., \& Carrera, F. J. 2001, Science, 294, 2516

Persson, S. E., Murphy, D. C., Krzeminski, W., Roth, M., \& Rieke, M. J. 1998, AJ, 116, 2475

Reichard, T. A., Richards, G. T., Hall, P. B., et al. 2003, AJ, 126, 2594

Richards, G. T., Hall, P. B., Vanden Berk, D. E., et al. 2003, AJ, 126, 1131

Ridgway, S. E., Heckman, T. M., Calzetti, D., \& Lehnert, M. 2001, ApJ, 550, 122

Sofia, U. J., Wolff, M. J., Rachford, B., et al. 2005, ApJ, 625, 167

Spergel, D. N., Verde, L., Peiris, H. V., et al. 2003, ApJS, 148, 175

Stocke, J. T., Morris, S. L., Weymann, R. J., \& Foltz, C. B. 1992, ApJ, 396, 487

Voit, G. M., Weymann, R. J., \& Korista, K. T. 1993, ApJ, 413, 95

Weymann, R. J., Morris, S. L., Foltz, C. B., \& Hewett, P. C. 1991, ApJ, 373,23

Willott, C. J., Rawlings, S., \& Grimes, J. A. 2003, ApJ, 598, 909

Zheng, W., Kriss, G. A., Telfer, R. C., Grimes, J. P., \& Davidsen, A. F. 1997, ApJ, 475, 469

Zuther, J., Eckart, A., Scharwächter, J., Krips, M., \& Straubmeier, C. 2004, A\&A, 414, 919 